Proceedings of the ASME 2020 Conference on Smart Materials, Adaptive Structures and Intelligent Systems

SMASIS2020

September 14-16, 2020, Irvine, CA, USA

SMASIS-2287

\title{
INVESTIGATION AND ATTEMPT TO 3D PRINT PIEZOELECTRIC 0-3 COMPOSITES MADE OF PHOTOPOLYMER RESINS AND PZT
}

\author{
Rytis Mitkus, Andreas Pierou, Julia Feder, Michael Sinapius \\ TU Braunschweig, Institute of Adaptronic and Function Integration \\ Braunschweig, Germany
}

\begin{abstract}
The present study demonstrates the manufacturing and characterization of 0-3 piezoelectric composites made of up to 10 vol\% of Lead Zirconate Titanate (PZT) particles and photopolymer resins. The tape-casting method was used to investigate the curing behavior, PZT loading limitations and the overall feasibility of the suspensions for $3 D$ printing. Piezoelectric composites were $3 D$ printed with a commercial DLP type $3 D$ printer. As a starting point, the maximum possible vol\% loading of PZT ceramic for each photopolymer resin was investigated. Five different commercially available photopolymer resins from Formlabs (Somerville, MA, US) were used. It was found that the addition of PZT particles to the photopolymer increases the time required for the photopolymer to solidify because PZT particles scatter the UV light. The approximate solidification time of each composition was measured, followed by viscosity measurements. SEM imaging of the composites showed good particle dispersion with minimum agglomeration, low particle sedimentation, but the weak bond between PZT particles and the photopolymers. Best performed material composition with 10 vol\% of PZT was used for $3 D$ printing. An attempt to shorten exposure time during printing was done by adding photoinitiator TPO. Suspensions with and without TPO were 3D printed and compared.
\end{abstract}

Keywords: 3D printing, piezoelectric composite, photopolymer resin, photoinitiator, TPO.

\section{INTRODUCTION}

Piezoelectricity is defined as the capability of certain materials to convert mechanical energy into electrical energy and vice versa. This ability received a great deal of attention over the last decades and finds applications in several fields such as structural health monitoring, energy harvesting, active noise control, and many others. The brittle nature of bulk piezoelectric ceramics in many cases limits the areas of their application. For these reasons, extensive research has been conducted for the development of flexible, two-phase piezoelectric composites made of polymer and ceramic, with the simplest form being the 0-3 composites. In this configuration, piezoceramic particles are usually dispersed in various polymers, such as epoxies or PVDF and forms a high viscosity suspension.

Some of the most common manufacturing methods for 0-3 composites are tape casting, compression molding, and spin coating. Typically these methods are labor-intensive, costly and time-consuming. As an example, epoxies require high temperatures and special conditions to solidify (heat and vacuum), where the equipment is costly, bulky and usually is found only in laboratories. Moreover, these manufacturing methods limit the achievable geometry of the composites, because composites are mostly cast in special forms or are cut in shape from bigger composite foils.

This study explores the possibility of using commercially available photopolymer resins instead of epoxies or polymers for the manufacturing of the 0-3 composites. It is believed that piezoelectric composites made of photopolymer can achieve the same or even higher piezoelectric outputs than piezoelectric composites made of other polymers or epoxies [1,2]. Photopolymer resins are a type of resins where polymerization is triggered with UV light and are most commonly used in Additive Manufacturing processes such as SLA and DLP [3]. Very complex geometries can be achieved by exposing a thin layer of photopolymer to UV-light, which is applied only in regions where needed. When photopolymer resins are exposed to UV light, photoinitiators break down into free radicals which in turn initiate the polymerization of monomers - solid body is built $[3,4]$.

The usage of photopolymers together with 3D printing for piezoelectric composite manufacturing reduces manufacturing time and the number of devices needed compared with the use of epoxies. Moreover, photopolymers enable the direct printing of 
piezoelectric composites with well-established AM processes such as SLA and DLP. Secondly, highly tailorable, applicationdependent composite properties can be achieved because various photopolymers with varying mechanical properties are commercially available. Thirdly, 3D printing could reduce manufacturing costs and time while at the same time could maintain high manufacturing accuracy. Furthermore, 3D printing of piezoelectric composites requires less labor and also reduces toxic material waste.

\section{BACKGROUND}

In the last years, a huge science community interest was shown in 3D printing of piezoelectric materials. Researchers already proved that both piezoelectric composites [1,2,5-9] and piezoelectric solid ceramics [10-16] could be directly 3D printed with the stereolithography process. 3D printing provides a geometrical degree of freedom, which with proper designing [2] and chemical modification methods [7,9] allowing achieve extremely enhanced piezoelectric properties of flexible, 3D printed composites $[1,7,9]$. However, further understanding is still required to fully master the highly complex stereolithography process of suspensions made of photopolymers and ceramic inclusions.

In recent years, multiple reviews on stereolithography of ceramics were published that are the perfect base for a deeper investigation of highly complex ceramic stereolithography process $[4,17,18]$. The main factors that influence $3 \mathrm{D}$ printing of piezoelectric composites are the chemical composition of the photopolymer, its viscosity, refractive index of materials, UV light intensity, exposure time and the loading of ceramic inclusions [6,17]. UV light intensity and exposure times are machine-dependent. The exposure time can be changed and usually can be used to counter low light intensity. However, chemical composition and viscosity are more important. For the best printing results, suspensions with low viscosity are advantageous, when using commercial 3D printers because the recoating process is less problematic and thinner layers can be printed [6]. For the highest piezoelectric performance as high piezoelectric ceramic loadings as possible should be used [7] but this increases the viscosity of the suspension.

The literature already proved that ceramic additives significantly increase the viscosity of the suspension [5] and undergo a sharp rise in viscosity at higher ceramic loadings $(>30$ vol\% of PZT, particle size $5 \mu \mathrm{m})[6,16]$. Moreover, the ceramic inclusions block, scatter and absorb the UV-light [11] that in turn decreases printable layer thickness, printing resolution [19] and requires higher light intensity and exposure time to succeed in printing $[4,6,17,19]$. It was reported, that scattering and absorption of the UV-light by ceramic particles are mainly influenced by the refractive index and particle size of the ceramic inclusions $[4,11,17]$ where the cure depth is inversely proportional to the square of the refractive index difference between ceramic inclusions and the photopolymer [20]. Badev et al. [21] concluded that bigger ceramic particles produce even bigger light scattering that makes the curing process even more problematic. Various PZT particle sizes, ranging from $5 \mu \mathrm{m}$ [6] to as low as $220 \mathrm{~nm}$ [2] were investigated by the researchers at different ceramic loadings. Most researchers added up to 50 vol\% of PZT [1,10,16] and succeeded in 3D printing of such suspensions. However, in most studies, researchers reported increasing the exposure time and UV-light intensity and at the same time 3D print thinner layers $[5,6]$ or had to modify their machines to successfully $3 \mathrm{D}$ print high viscosity suspensions $[1,2,8]$.

This research examines the suitability of 5 different commercially available photopolymers for the 3D printing (SLA and DLP) of 0-3 piezoelectric composites made of photopolymers and PZT particles. Commercially obtainable photopolymers were chosen for their availability. It is expected that the differences between chemical, mechanical and thermal material properties will result in varying piezoelectric performance.

\section{PIEZOELECTRIC COMPOSITES BASED ON PHOTOPOLYMERS}

Experiments were conducted to investigate the suitability of the photopolymers for 3D printing of piezoelectric composites. Firstly, the tape-casting method was used to investigate the curability of the photopolymers when ceramic particles are added. Secondly, the piezoelectric composites were manufactured by tape-casting, electroded and polarised to prove their piezoelectric output. Thirdly, the composites were 3D printed with a 3D printer. Finally, an attempt to add additional photoinitiators to decrease exposure time required during the $3 \mathrm{D}$ printing process is presented.

Five different, photopolymer resins commercially available from Formlabs were investigated (Clear V4, Flexible V2, Durable V2, High-Temperature V1, and Tough V4). These resins are characterized by their different viscosity in liquid form, and different mechanical and thermal properties in their solid form. As a piezoelectric ceramic filler, PZT particles (PIC225, average size 1,6 $\mu \mathrm{m}$, PI Ceramic, Germany) were used.

For experiments, a customized UV-lamp was used, which is made of the LED light strip (5 m in length with 150 LEDs, $24 \mathrm{~W}$, a wavelength of $395 \mathrm{~nm}, \mathrm{UV}-30$, Renkforce). The area of light is $0,05 \mathrm{~m}^{2}$. While the ideal curing wavelength for the photopolymers used is $405 \mathrm{~nm}$, it is expected that a $10 \mathrm{~nm}$ deviation will not have a significant influence on the polymerization process and subsequently the mechanical properties [22].

\subsection{Tape-casting of piezoelectric composites}

As a preparatory experiment, the five photopolymer resins were tape-cast without PZT particles as a reference on a glass plate with a blade at 45 degrees. Metal stripes were used to set layer thickness of $0,4 \mathrm{~mm}$ which is quite thick. Lower casting thicknesses are problematic, because of the low viscosity of the photopolymers. They tend to form droplets instead of a thin layer while being cast on the glass. After casting, the UV-lamp was placed on top of the cast specimens at a distance of $10 \mathrm{~cm}$.

All five casted photopolymers were successfully cured with the UV-lamp in 2 minutes and had a thickness of $0,25 \mathrm{~mm}( \pm 0,05$ 


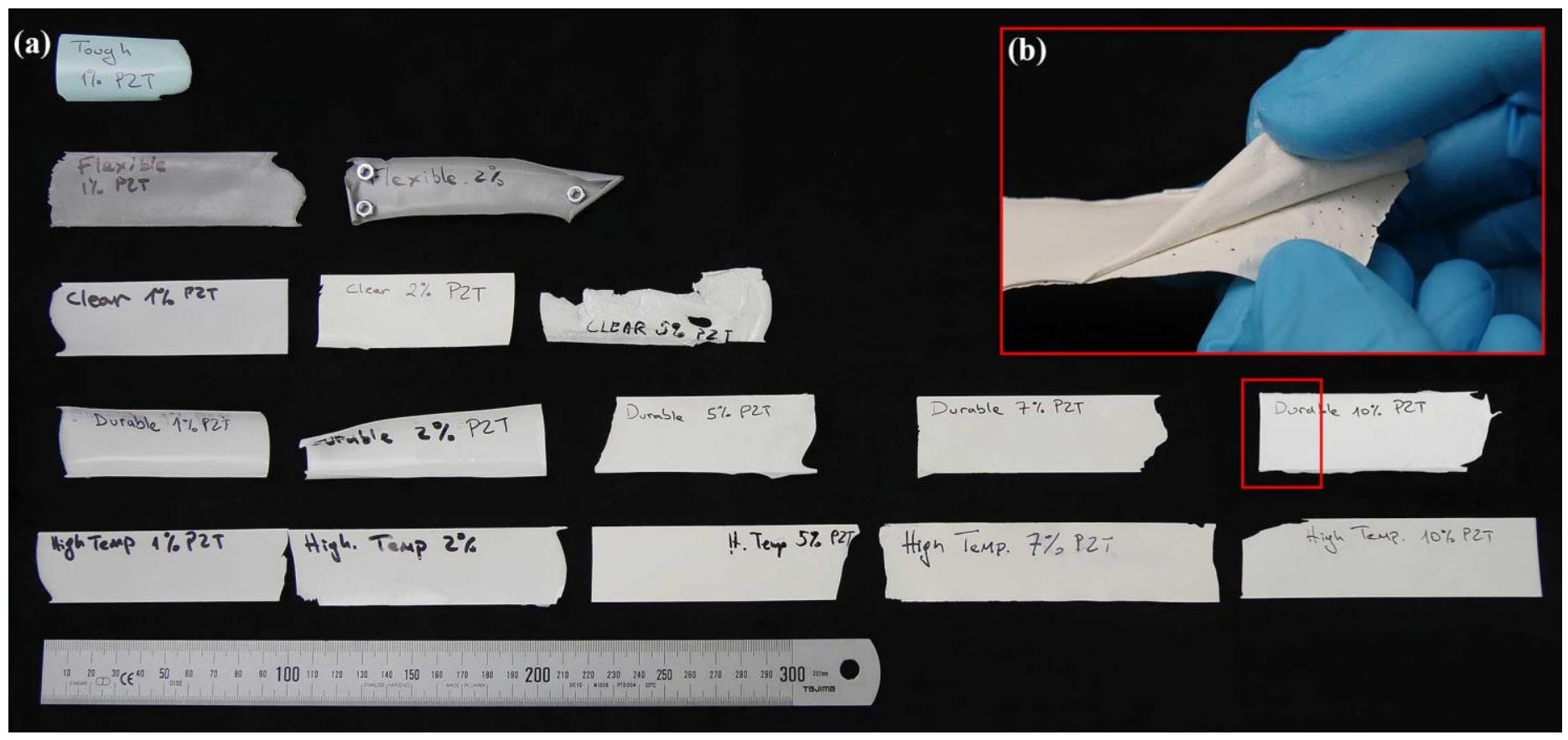

Figure 1. Tape-casted piezoelectric composites with varying PZT concentration. a) Different photopolymers used, starting from the top: "Tough", "Flexible", "Clear", "Durable", "High-Temperature"; b) Observed layer separation in higher PZT concentration composites.

mm). A simple wooden stick was used to scratch the surface of the casted suspensions to evaluate if the casted layer is solid. After the successful preparatory experiments, PZT volume influence on the curability of the suspensions was investigated to find the maximum possible PZT particle content. Since piezoelectric ceramic particles are the active phase in the composites, their loading has a direct influence on the piezoelectric output of the composites. Finding the maximum possible loading percentage that still allows a suspension to solidify under UV-light is the first most important step. Photopolymers used in this study are not meant for being used with ceramic fillers added to them. For this reason, low loading percentages were expected. On the other hand, PZT loadings up to $50 \mathrm{vol} \%$ were reported in the literature $[1,10,16]$.

The experiment started by adding 1 vol\% of PZT to the five different photopolymers because thick layers were planned to be cured. PZT particles and photopolymer resin were mixed in a centrifugal mixer for 3 minutes under constant vacuum. The mixed suspensions consisting of photopolymers and PZT were poured on the glass plate and tape-casted with a blade at 45 degrees. After a maximum of 1 hour, the cured composites were peeled off the glass plate and were washed manually with Isopropyl alcohol. Mixing parameters, casting thickness and curing parameters were kept constant through-out the experiments in this study.

PZT loading was gradually increased until only two the most promising photopolymers left, which successfully cured with 10 vol\% PZT loadings. Uncured compositions were discarded from further experimentation. The curing stage of the suspension was characterized by a simple wooden stick, which was used to scratch the surface of the casted suspensions every 10 minutes. A scale from 0 to 5 was set to examine the curing stage: 0 wooden stick can fully penetrate the whole cast suspension layer and touch the glass; 5 - the suspension is fully cured and it is impossible to scratch the surface. Regardless of the error margin that this method allows, valuable information was extracted regarding the relationship between loading percentages, curing time and resin curing characteristics.

\subsection{Results of tape-casting}

Each photopolymer exhibited a saturation point at different ceramic loading percentages. Experiments were repeated with up to $10 \mathrm{vol} \%$ of PZT where only Durable and High-Temperature photopolymer resins cured successfully. Figure 1a represents all composites manufactured in our study with tape-casting at different PZT particle loadings. Figure 2 represents the curing characteristics of Durable and High-Temperature photopolymers. With all the composites manufactured, "Tough" showed the worst result and only 1 vol\% loading was successfully solidified (Fig. 1a, first specimen from the top). "Flexible" and "Clear" followed with loading limits at 2 and 5 vol\% respectively (Fig. 1a, second and third specimens from the top respectively). Promising results were exhibited only for "Durable" and "High Temperature" photopolymers. These two photopolymers cured with up to $10 \%$ vol of PZT. Moreover, High Temperature and Durable were also investigated with 15 vol\% PZT loadings. Composites manufactured at these 


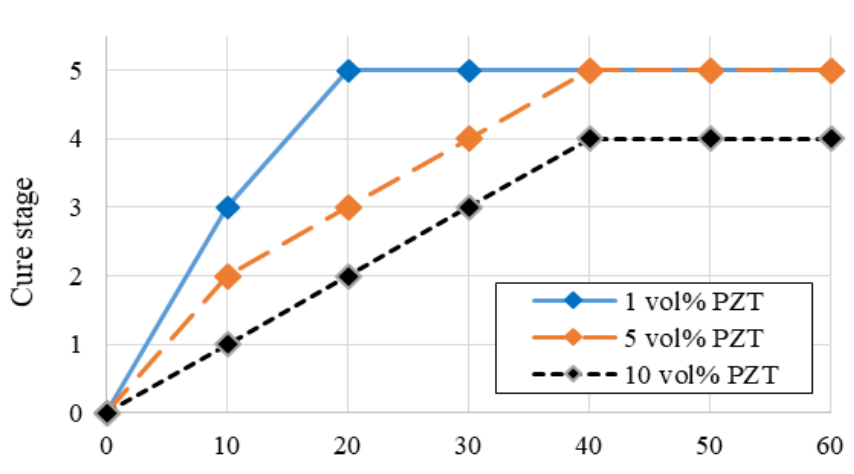

(a)

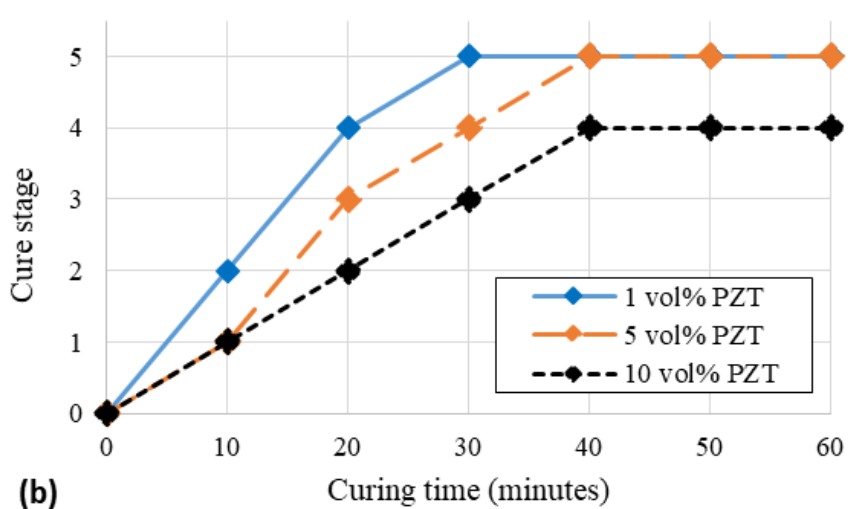

Figure 2. Cure stage as a function of curing time for different PZT concentrations in: (a) High-Temperature photopolymer resin; (b) Durable photopolymer resin.

percentages did not fully cure and soft, gel-like consistency was achieved after 1 hour of curing. They are not added to Figure 1.

The different behavior of each photopolymer under the same curing conditions can be interpreted as the difference in photopolymer refractive index and different chemical composition of the photopolymers [4]. Unfortunately, the manufacturer of the investigated photopolymers does not provide the exact chemical composition or refractive index of each material so that no general conclusion can be drawn.

As it was expected, a correlation could be observed between loading percentages and curing time. Figure 2 represents the correlation between PZT loading and the curing time of two photopolymers used. As the ceramic loading increases, longer exposure times are required for the suspensions to fully cure. This is exactly as expected because ceramic inclusions reflect most of the UV light.

If we consider as a first example High-Temperature photopolymer (Figure 2, left), we can see that cure stage 5 is reached within 20 minutes when only $1 \mathrm{vol} \%$ of PZT is used and as loading increases to $5 \mathrm{vol} \%$ of PZT, curing stage 5 is delayed to 40 minutes. The same tendency is also observed for Durable (Figure 2, right) where at $1 \%$ vol of PZT cure stage 5 was reached at 30 minutes and for $5 \mathrm{vol} \%$ at 40 minutes. For both suspensions curing slope angle drops and longer exposure times are required with the increase of the ceramic phase. For $10 \mathrm{vol} \%$, none of the suspensions managed to reach stage 5 within 1 hour. This was especially noticeable while washing the composites with isopropanol alcohol where for these composites some of the uncured suspension together with some PZT particles was washed off of the top surface.

If we consider High-Temperature composites again for comparison, the uncured suspension on the surface appeared at loading percentages starting at $7 \mathrm{vol} \%$ with the increase of this effect as ceramic loading increased. This effect appeared with the composites made of photopolymer "Clear" already at $1 \mathrm{vol} \%$. Loose suspension on the top surface indicates that a higher intensity of UV-light or even longer exposure times are required to solidify that composition.

Contrary to expectations it was observed that all suspensions started to cure from the bottom (glass surface) upwards, but at the same time, some polymerization started to occur on the top surface. This lead to some composites, especially at higher ceramic loadings ( $7 \%$ vol of PZT), that had thin, uncured suspension layer in between two fully cured top and bottom layers (see Fig. 1b). Casting at thinner layers $(0,2 \mathrm{~mm})$ seemed to minimize this effect to some extent, but the casting process was complicated.

It is believed that the polymerization at the bottom of the composite started because of the thick glass that was used. The glass, on which suspensions were cast, was $10 \mathrm{~mm}$ thick. It is suspected that some light penetrating through a glass was reflected inside the glass and started to cure the cast suspension from the bottom.

Moreover, it is believed that the thin layer of uncured suspension in the middle of the specimens could be explained by a thick cast layer that had $0,4 \mathrm{~mm}$ in thickness. When the polymerization starts, the viscosity of the photopolymer slowly increases and free radicals, which help to solidify the polymer, lose their mobility - photopolymerization process stops [23]. This is exactly what happened from both sides of the cast composite. After certain curing time, both surfaces were fully cured up to some depth and no further polymerization was able to happen deeper in the suspension - the thin layer of the suspension between the solid layers was not fully cured.

From results, it is clear that tape-casting has its limitations such as insufficiently characterized curing stage, light reflection by the glass and limited casting thickness which subsequently led to the two-layer separation effect. While a higher viscosity photopolymers might work better, the whole setup is highly dependent on the viscosity of the suspension. However, reliable data was extracted and both High-Temperature and Durable composites with 10 vol\% of PZT were investigated further.

\subsection{Viscosity measurements}

The low viscosity of the suspensions in liquid form is essential for successful 3D printing. Firstly, self-leveling of the suspension (viscosity <3 Pa.s) is beneficial in the printing process and reduces recoating problems [6] - suspensions can be used in commercial machines without any modifications. Furthermore, higher viscosity makes it more complicated for the printer to achieve very thin layers $(<50 \mu \mathrm{m})$ because the $\mathrm{Z}$-axis 


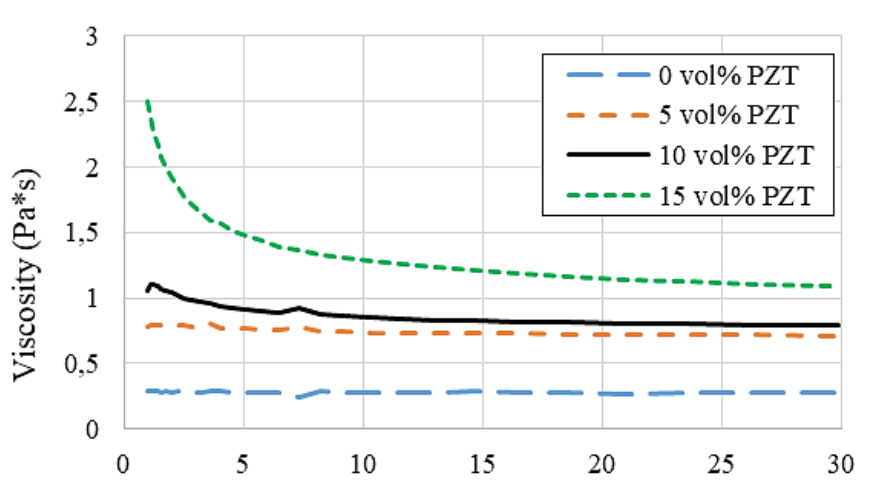

(a) Shear rate $(1 / \mathrm{s})$

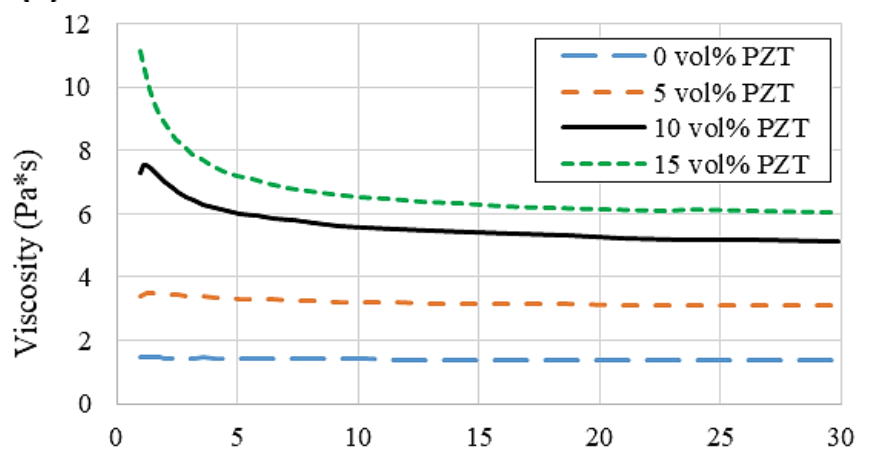

(b)

Shear rate $(1 / \mathrm{s})$

Figure 3. The viscosity of the suspension as a function of shear rate for different PZT concentrations in: (a) High-Temperature photopolymer resin; (b) Durable photopolymer resin.

of the printers is usually not strong enough to push the build plate and squeeze the photopolymer with ceramic inclusions to physically achieve the desired thickness of the layer. For these reasons, viscosities of all used slurries were measured at room temperature with a parallel plate Advanced Rheometer Germin, (BOHLIN Industries, Germany) and are shown in Fig. 3. The viscosities of the photopolymers without any ceramic particles are also added. In literature, it was reported that viscosity of 4.6 Pa.s at $50 \mathrm{~s}^{-1}$ is quite high for stereolithography [6] and material viscosity should be up to 3.0 Pa.s.

Resins at their pure form show Newtonian behavior (High Temperature has 0.8 Pa.s. and Durable 1.5 Pa.s) with an increase of viscosity as ceramic loading increases. At solid loading of 10 vol\% of PZT and higher, shear-thinning effects appear which leads to significant viscosity increase at low shear rates. Shearthinning effects at high shear rates can be utilized for recoating purposes in different AM processes since low coating forces will be required.

Concluding, at any given ceramic loading, a HighTemperature photopolymer has approximately six times lower viscosity than a Durable photopolymer. Lower viscosity is almost always better for 3D printing purposes because it helps to achieve thinner layers of the suspension being printed. Moreover, the results show that loadings up to $15 \mathrm{vol} \%$ PZT in a
High-Temperature photopolymer would be suitable for most commercial machines.

\subsection{SEM Imaging}

The particle distribution in cured composites was studied using SEM imaging. Figure 4 shows the cross-sections of both High-Temperature and Durable piezoelectric composites with 10 vol\% of PZT loadings. For SEM imaging, the composites were broken and were sputtered with a very thin layer of platinum.

SEM revealed no significant differences in particle dispersion between High Temperature and Durable composites. Homogenous particle distribution with no significant signs of agglomeration was observed in both composites. Regardless of the expectations, no particle sedimentation was observed which is believed to be a result of rapid polymerization under UV light. The mixed suspensions were cast and cured in one hour directly after mixing. This is another advantage of using photopolymers instead of epoxies because photopolymers can be cured very fast and particle sedimentation can be minimized as opposite to composites made of epoxies that mostly require curing of hours in a temperature-controlled environment. However, it is believed that storing mixed suspensions for an extended period would lead to significant particle sedimentation because the density of PZT particles $\left(7,8 \mathrm{~g} / \mathrm{cm}^{3}\right)$ is almost 7 times higher than the average density of photopolymers used in this study $(1,14$ $\left.\mathrm{g} / \mathrm{cm}^{3}\right)$.

In the SEM images, it is observed that ceramic inclusions in High-Temperature specimens do not form any bond with photopolymers, because some particles dropped away when composite was broken for SEM imaging. Almost no bond between ceramic inclusions and the High-Temperature photopolymer can also be proven because small gaps between PZT particles and photopolymer can be seen around some ceramic particles. These gaps probably appeared during the breaking of the composite, where ceramic particles slightly moved from their positions in the composite. On the other hand, the Durable photopolymer shows stronger bonds with ceramic inclusions, because only a few missing ceramic particles can be observed in the SEM image. Moreover, almost no gaps between ceramic particles and photopolymers are observable. The stiffness of the photopolymers might influence the results because the Durable composite has lower stiffness which leads to lower stresses during the breaking of the composite for SEM imaging and thus particles stay in the composite and do not fall away.

Furthermore, very small porosity can be observed in the SEM images in both composites, but it is unclear if it is air gaps introduced during the manufacturing process or moved/fallen particles during breaking processes that look like air gaps in the SEM images.

\subsection{Quality characterization}

Both High-Temperature and Durable piezoelectric composites with 10 vol\% of PZT were tape-cast, cured under UV-light, cut in shape $(30 \times 30 \mathrm{~mm})$, electroded and polarised with $20 \mathrm{kV} / \mathrm{mm}$ in Silica oil under room temperature for 20 

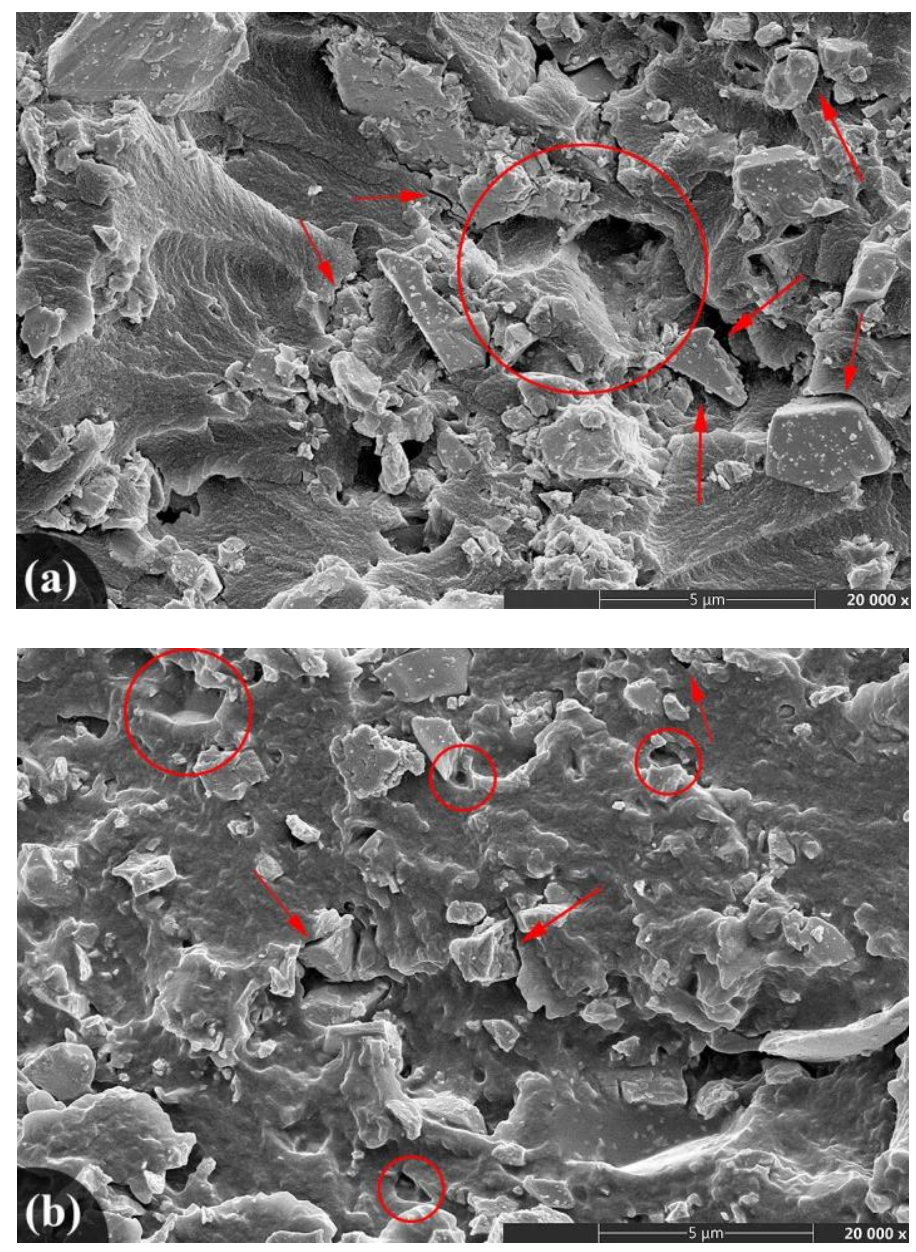

Figure 4. SEM images of cross-sections of 10 vol\% PZT in: (a) High-Temperature photopolymer resin; (b) Durable photopolymer resin.

minutes to investigate the quality of their manufacturing and their usability as piezoelectric composites. The electrodes of 100 $\mathrm{nm}$ thickness were gold-sputtered on both sides of the specimens. The composites are shown in Figure 5a-b. It cannot be seen from the image but the top surfaces of the composites (Figure 5a-b) are somehow rough (varying electrode color), compared with the bottom sides which were cast directly on the glass surface and are very even. During the polarisation process, some composites experienced a breakdown. It could indicate slight porosity in the composite or irregularities in its manufacturing. On the other hand, it can also indicate the maximum polarisation voltage possible for such composites.

Figure 5c-d shows the flexibility of manufactured composites with 10 vol\% of PZT particles. Because of the higher stiffness of the photopolymer, High-Temperature composite exhibits lower flexibility (Figure 5c) compared with Durable composite (Figure 5d). Higher deformation of the HighTemperature composite leads to a broken composite.

\section{PRINTING OF PIEZOELECTRIC COMPOSITES}

A suspension made of High-Temperature and $10 \mathrm{vol} \%$ of PZT ceramic was selected for 3D printing because of its lower viscosity. The same mixing procedure as in the previous chapter was used. The aim was to $3 \mathrm{D}$ print the specimen with a minimum of $200 \mu \mathrm{m}$ in thickness because the composites thinner than 200 $\mu \mathrm{m}$ are usually too flexible and not suitable for our polarisation device. On the other hand, the maximum printable layer height in SLA and DLP systems usually is $100 \mu \mathrm{m}$, so the specimen will require a minimum of 2 layers to print, thus thicker structures are more suitable as printing test specimens.

Two different 3D printers were used: Formlabs Form-2 (SLA) and Prusa SL1 (DLP). The Form-2 SLA-type printer is not open-source and only the manufacturer default print settings can be used. Unfortunately, it is only possible to choose material type and layer thickness. The printer uses $250 \mathrm{~mW}$ laser with a laser spot of $140 \mu \mathrm{m}$ and a $405 \mathrm{~nm}$ wavelength. Resin tank preheating, material refilling and material mixing functions (wiper) are automatically disabled because the printer must be used in "Open-mode". By choosing different material printing settings, light intensity and exposure times are changed. However, any exact values of light intensity or exposure time are unknown.

Extensive attempts revealed that it is impossible to 3D print piezoelectric composite with Formlabs Form-2 printer. A single layer was successfully solidified but it was found on the bottom of the resin tank. By adjusting the first layer height (through a gap between the build platform and resin tank) and testing multiple possible print setting configurations (46 combinations in total), the maximum thickness of the composite of $110 \mu \mathrm{m}$ was

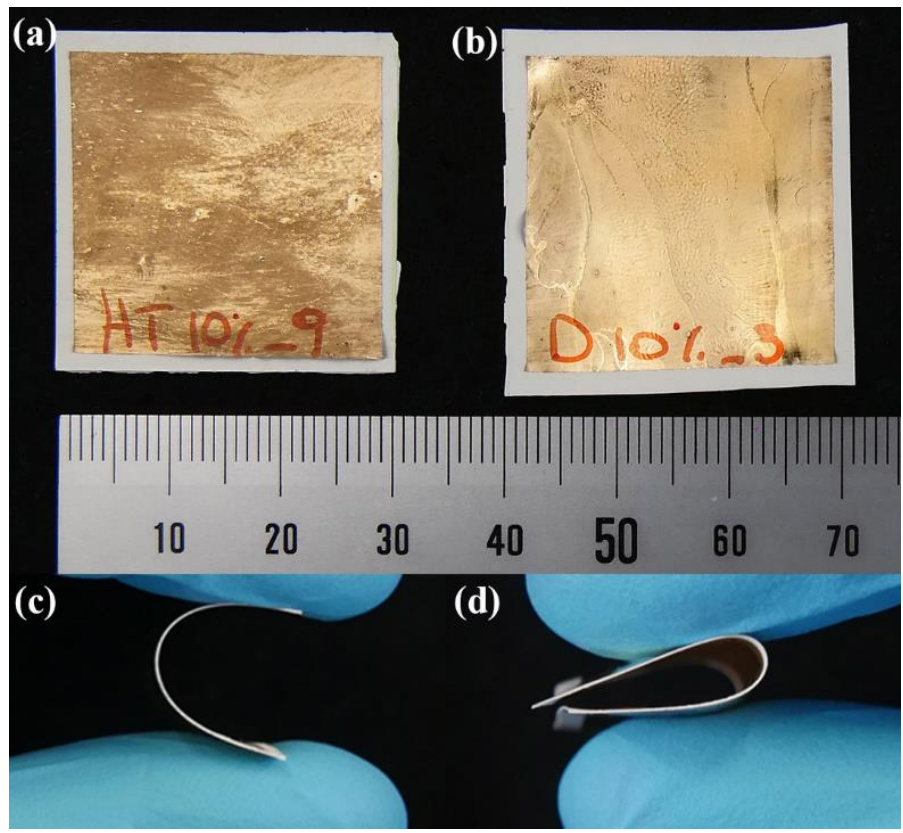

Figure 5. Tape-cast, cleaned, cut, electroded and polarised piezoelectric composites. (a) High-Temperature; (b) Durable; (c) Flexibility of High-Temperature piezoelectric composite; (d) Flexibility of Durable piezoelectric composite. 
achieved using the print settings "Black V1" with layer height set to $50 \mu \mathrm{m}$. Unfortunately, the printed specimen was also found on the bottom of the resin tank again. No adhesion to the build plate was achieved. Because the specimen does not stick to the build platform it is impossible to print consecutive layers. Although it is clear that the ceramic inclusions absorb/scatter the UV-light and higher exposure times are required, this printer turns out to be not suitable for our research purposes because of a lack of settings that could adjust the light intensity, layer height and/or exposure time.

On the other hand, Prusa SL1, a DLP-type 3D printer is an open-source machine, which allows adjusting the layer exposure time and change layer thickness. Almost any photopolymer suitable for photopolymerization can be used with this printer. Prusa SL1 uses an LED UV-light source that produces UV-light intensity of $1-1.2 \mathrm{~W} / \mathrm{m}^{2}$ according to the manufacturer. $2 \mathrm{~K}$ resolution LCD screen is used to control the exposure of the build platform. It has a high XY-resolution of $0.047 \mathrm{~mm}$ per pixel.

3D printing of piezoelectric composites was successful with the Prusa SL1 printer. The composites with a thickness of 0,2 $\mathrm{mm}$, made of 3 layers were successfully 3D printed. The first layer had a thickness of $120 \mu \mathrm{m}$ and each consecutive layer was about $45-50 \mu \mathrm{m}$ in thickness. The main problem was to ensure that the specimen being printed sticks to the build platform so that multiple layers could be printed. From previous experiments with another printer, it is clear that the adhesion of the first layer to the build platform is always a challenge. It is believed that adhesion was poor because of too short exposure time or too thick printed layer. To increase adhesion, either exposure time must be increased or layer thickness must be decreased or both. Researchers report in the literature that the cure depth should be twice the layer height to ensure polymerization between two layers [2]. The cure depth is mainly controlled with the exposure time and light intensity. From literature it is also known that thinner layers require shorter exposure times, thus makes the printing process faster [4]. In our study, ceramic inclusions scatter UV-light and decrease the cure depth. At the same time, this scattered light makes UV-light exposed spot wider, thus printing resolution is reduced [19]. Thinner layers increase the resolution of printing while requiring shorter exposure time for curing.

Starting with a default first layer height of $50 \mu \mathrm{m}$, the height was incrementally decreased from $50 \mu \mathrm{m}$ to $20 \mu \mathrm{m}$. At the same time, different exposure times (minutes) have been also investigated. The print was successful at $20 \mu \mathrm{m}$ layer height and 12 minutes exposure time. However, the printed single layer of the piezoelectric specimen was still found on the bottom of the resin tank and had a thickness of $110 \mu \mathrm{m}$ which is much thicker than the set value of $20 \mu \mathrm{m}$.

When the layer height of the first layer was set to $20 \mu \mathrm{m}$ in the slicing software, it was noticed that the printer was not able to reach this set of $20 \mu \mathrm{m}$ value physically because of the increased viscosity of the suspension. It was observed that the whole frame of the printer bends slightly when the build platform squeezes the suspension with ceramic particles to achieve the first layer height. For this reason, the physical first layer height was decreased further by adjusting the offset of the first layer directly on the printer to $-0,150 \mu \mathrm{m}$. In this case higher force is applied on the build platform to squeeze high viscosity suspension to make a thin layer. Now, the print was successful and the printed specimen stuck to the build platform. Surprisingly, the layer was $120 \mu \mathrm{m}$ thick - even thicker than before. The reason for this is not yet clear because exposure time and layer thickness were kept constant, only the first layer offset value was decreased, which was supposed to make the layer thinner. Twelve minutes were needed to cure the first layer. When the photopolymer without any ceramic inclusions is used, the printer requires about 30 seconds to cure the first layer that usually has a thickness of $50 \mu \mathrm{m}$ and requires about 6 seconds to cure consecutive layers. Longer exposure time for the first layer is usually used to ensure adhesion to the build platform. In this study, for all 3 printed layers of piezoelectric composite, 12 minutes exposure time was used, but it is believed that for the second and next layers, lower exposure times could be sufficient.

The ceramic inclusions in the photopolymer increase the viscosity of the suspension. In turn, higher viscosity requires higher forces to create a very thin layer of the suspension. Unfortunately, the printer without any modifications is not able to achieve the first layer height of $20 \mu \mathrm{m}$ as was set in the slicing software. Another adjustment that might help to physically achieve a thin first layer could be a very slow build platform lowering. However, while Prusa SL1 has a slow build platform lowering option, the speed of it cannot be adjusted. On the other hand, second and third printed layers seem to have 40-50 $\mu \mathrm{m}$ layer heights as was set in slicing software, most likely due to the reduction of the surface area in the consecutive layers that are in contact with the resin tank. When the printer lowers the build platform for the first layer, the contact area is approximately $9360 \mathrm{~mm}^{2}$, however, for the second or third layer the contact area in our experiments was only $1800 \mathrm{~mm}^{2}$ because only 2 specimens were printed at the time. For this reason, the forces required to squeeze the suspension to $50 \mu \mathrm{m}$ layer are lower and the printed does not have any problems achieving set layer thickness for second, third and next layers.

In summary, a suspension made of High-Temperature photopolymer and 10 vol\% of PZT can be successfully used to 3D print piezoelectric composites on commercially available Prusa SL1 printer. However, printing parameters to ensure the first layer adhesion to build a platform must be experimentally found. It was experimentally found that low layer height in printing settings, negative offset adjustment and long exposure time ensures the adhesion of the first layer to the build platform.

\section{ADDITION OF PHOTOINITIATOR}

As the next step after the successful 3D printing of the piezoelectric composites, an attempt was done to reduce the exposure time required for the composites by the addition of a photoinitiator. The well-known trimethyl-benzoyl-diphenylphosphine oxide (TPO) photoinitiator, which has already been used in several studies [23,24], was selected. TPO has an absorption spectrum of $320-410 \mathrm{~nm}$, which matches the 


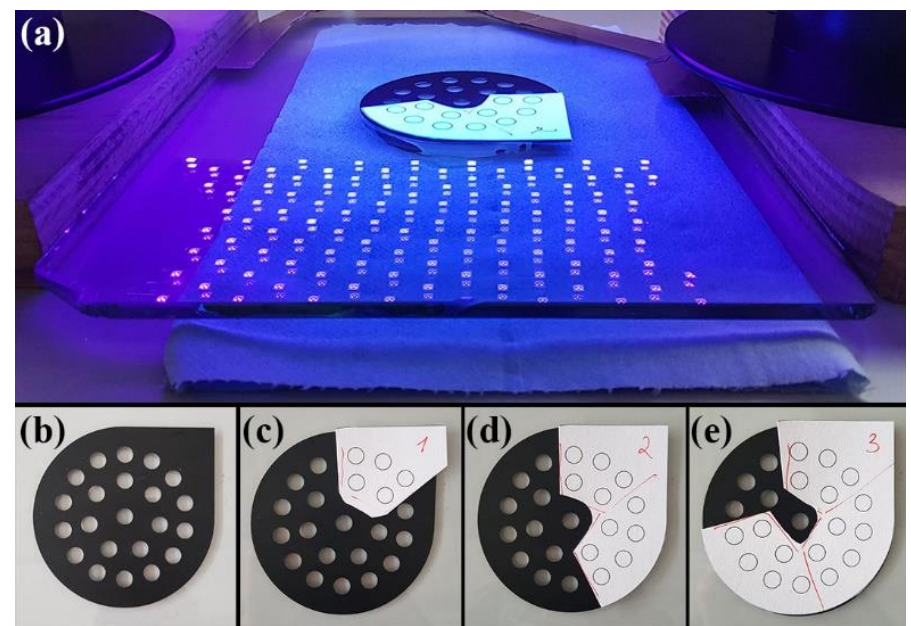

Figure 6. Curing setup. (a) Glass with the mask placed on the container with the suspension; (b) No mask at $\mathrm{t}=0 \mathrm{~min}$; (c) Mask at $\mathrm{t}=2 \mathrm{~min}$; (d) Mask at $\mathrm{t}=4 \mathrm{~min}$; (e) Mask at $\mathrm{t}=6 \mathrm{~min}$.

wavelength used by the printer $(405 \mathrm{~nm})$. The main aim was to investigate how the addition of TPO influences the curing depth and overall print quality of piezoelectric composite. Higher curing depth reduces exposure time required, thus makes 3D printing faster. In the literature, the best results are reported at $1.18 \mathrm{wt} \%$ concentration of TPO [23]. Therefore it is assumed that a saturation point of TPO exists, where further addition of photoinitiator would negatively influence the cure depth of the suspension.

To investigate the TPO influence on the cure depth the same suspension as before was used: High-Temperate photopolymer with 10 vol\% PZT particles. According to the photopolymer material datasheet, our used High-Temperature material has already less than $1 \%$ (it is not written if it is wt\% or vol\%) of photoinitiator inside [25]. Unfortunately, the exact content is unknown. We added up to $4 \mathrm{wt} \%$ of TPO to the suspension with $0,5 \mathrm{wt} \%$ increments and acquired in total 9 different suspensions with varying TPO contents. The weight percentage of TPO was used instead of volumetric for easier comparison with other studies. Each suspension was exposed 2, 4, 6 and 8 minutes to UV light.

The experiment setup proposed by Bennett et al. [26] was used and was slightly modified to our equipment (Figure 6a). It consists of the container where the suspension is filled. On top, a glass plate with a mask is placed. During experiments, it was ensured that no air bubbles are trapped and that the suspension touches the whole area of interest on the glass. Mask used had a total of 22 holes with a diameter of $8 \mathrm{~mm}$ each and at least $5 \mathrm{~mm}$ between the holes (see Figure 6b). The maximum exposure time of 8 minutes was used. Different exposure times for specific holes were achieved by covering the respective holes with the masks as shown in Figure 6c-e. An experiment was started without any mask, and every 2 minutes the respective mask was used to cover respective holes from further exposure. This allowed us to investigate 4 different exposure times for every suspension used. UV-light source was positioned $7 \mathrm{~cm}$ above the glass.

After the exposure of a maximum of 8 minutes, the glass is removed from the container, is cleaned and is placed on two even metal blocks for measurement (see Figure 7a). The cure depth of every cured circle is measured with a laser distance sensor installed above. By sliding the glass on the calibrated metal blocks, the height of all cured circles was measured. The blue sticker with a known thickness on the corner was used as a reference point. The height of each circle was measured at 3-5 points and the highest value was recorded. The results obtained are presented in Figure $7 \mathrm{~b}-\mathrm{j}$. It is clear that at higher TPO contents over-exposure becomes clearly visible. It starts to appear at $0,5 \mathrm{wt} \%$ of TPO and increases with increased TPO
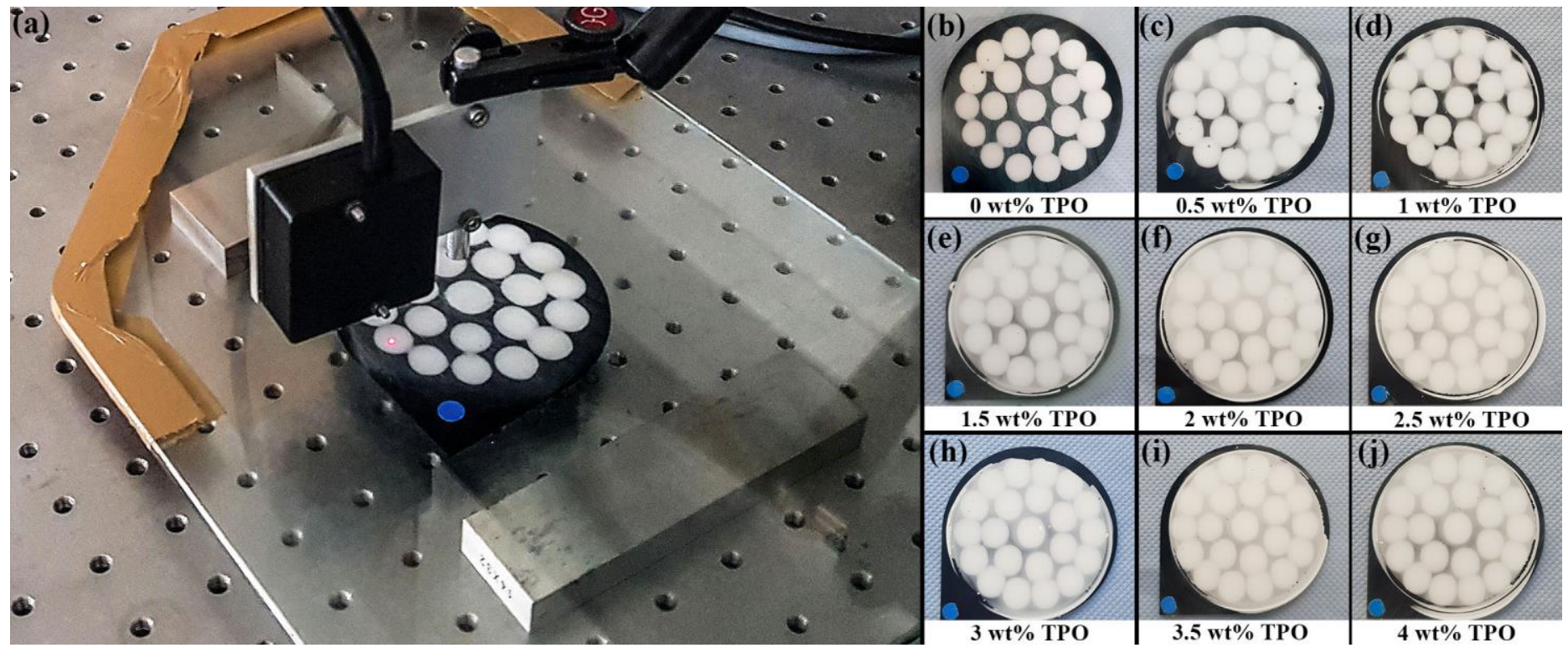

Figure 7. Cure depth measuring setup. a) Measuring setup; b) to j) Results. 


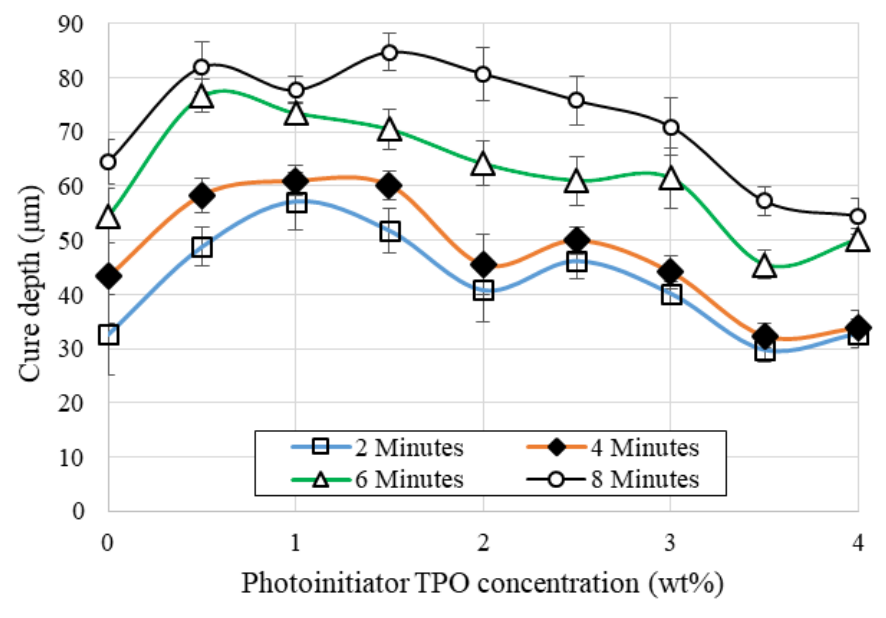

Figure 8: Cure depth as a function of photoinitiator wt $\%$ at different UV-light exposure times. Fillers content: 10 vol\% of PZT in High-Temperature photopolymer resin.

content because PZT scatters the light and scattered light hits photoinitiator particles in the suspension that is not under the light source. UV doping could be used as a method to absorb some of the free radicals [19], thus the over-exposure is reduced but at the same time cure depth also decreases. However, further experiments must be done, because it might be that at specific concentrations of photoinitiator and UV absorber only slightly reduced cure depth but strongly reduced over-exposure could be achieved.

Because of mistake during mixing, the suspension with 1 wt $\%$ of TPO was mixed another day. Unfortunately, the results of $1 \mathrm{wt} \%$ of TPO are not reliable, because from Figure $7 \mathrm{c}$ and $\mathrm{d}$ can be seen that suspension with $0,5 \mathrm{wt} \%$ TPO has higher overexposure (almost no black spaces are visible) than the suspension with 1 wt $\%$ of TPO. Most likely, TPO did not dissolve completely as it did in other investigated suspensions.

Figure 8 presents the cure depth as a function of photoinitiator concentration in weight at different UV-light exposure times. Each line represents a different exposure time. The results in Figure 8 also show lower values for $1 \mathrm{wt} \%$ TPO suspension than expected. Results reported by Dufaud et al. [6] lead to the expectation that at $1 \mathrm{wt} \%$ of TPO the peak of cure depth will be reached. At least the same cure depth as for 0,5 $\mathrm{wt} \%$ and 1,5 wt\% TPO was expected, but, much lower values were measured, especially for longer exposure times. Lower results than expected were recorded for $2 \mathrm{wt} \%$ of TPO as well at exposure times of 2 and 4 minutes (see Figure 8). It is believed that it might be a slight measurement error. Furthermore, the poor dissolving of TPO was noticed for the highest TPO loadings (3,5 and $4 \% \mathrm{wt}$ ) and the suspensions were re-mixed before the experiment.

In Figure 8 it can be seen that the mean layer thickness increases for all mixtures with increasing exposure time. However, a bigger increase can be noticed from 4 to 6 minutes of exposure time. The reason is still not clear. The maximum cure depth of $53.6 \mu \mathrm{m}$ after 2 minutes of exposure time is reached by the mixture containing $1.0 \mathrm{wt}$. \% TPO. On the other hand, the maximum cure depth of $84.75 \mu \mathrm{m}$ after 8 minutes of exposure time is reached by the mixture containing $1.5 \mathrm{wt}$ \% TPO. The increase of cure depth when increasing the exposure time can be explained by the bigger amount of radicals inside the mixture when being exposed to UV-light for a longer time [27]. The increase of free radicals increases the speed of the polymerization process and therefore cures a thicker layer.

In Figure 8 it can also be seen that the mean layer thickness decreases for amounts of TPO higher than $1.5 \mathrm{wt}$ \%. The same trend is reported by Dufaud et al. [6]. It is assumed that this is due to the faster polymerization process. The viscosity of the mixture increases with ongoing polymerization process [27]. For this reason, the movements of radicals and monomers inside the mixture are limited, which stops the polymerization process before a thicker layer can be cured [28].

The addition of TPO helps to increase the cure depth until the threshold of TPO is reached. In our case, it was around 0,5-1,5 wt\% of TPO. However, the used High-Temperature photopolymer already had less than $1 \%$ of unknown photoinitiators inside. Regardless of that, 3D printing of suspension with $1,5 \mathrm{wt} \%$ of TPO was done.

\subsection{Comparison of the piezoelectric composites with and without photoinitiator}

Suspension made of High-Temperature photopolymer, 10 vol\% of PZT and 1,5 wt $\%$ of TPO (wt $\%$ from the suspension) was successfully 3D printed. Starting with 12 minutes of exposure time, it was decreased to 5 minutes with 1 -minute decrements. Lower exposure times lead to adhesion problems of the first layer. 3D printing revealed that indeed shorter layer exposure times (5 minutes) can be used for a suspension containing 1,5 wt $\%$ of TPO compared to the exposure time required for a suspension containing no TPO (12 minutes). However, all printed composites with TPO, at any exposure times used, were over-exposed, thus the dimensions of the specimens were increased. Figure 9 shows 3D printed specimens with and without TPO.

\section{SUMMARIZING DISCUSSION}

Five different photopolymers from the company Formlabs were experimentally investigated for piezoelectric composite manufacturing suitability. A simple tape-casting method was used to test material suitability. Up to $10 \mathrm{vol} \%$ of PZT was successfully added to only two of the investigated materials: Durable and High-Temperature. However, our used tape-casting method seems to be not suitable for such characterization. Viscosity measurements of the suspensions showed an increase in viscosity of all investigated suspensions when ceramic inclusions are added. Due to the high viscosity with $10 \mathrm{vol} \%$ of PZT, a Durable photopolymer was discarded from further examination and only a High-Temperature photopolymer was used throughout our study. SEM images revealed no agglomeration or sedimentation in specimens made of High- 


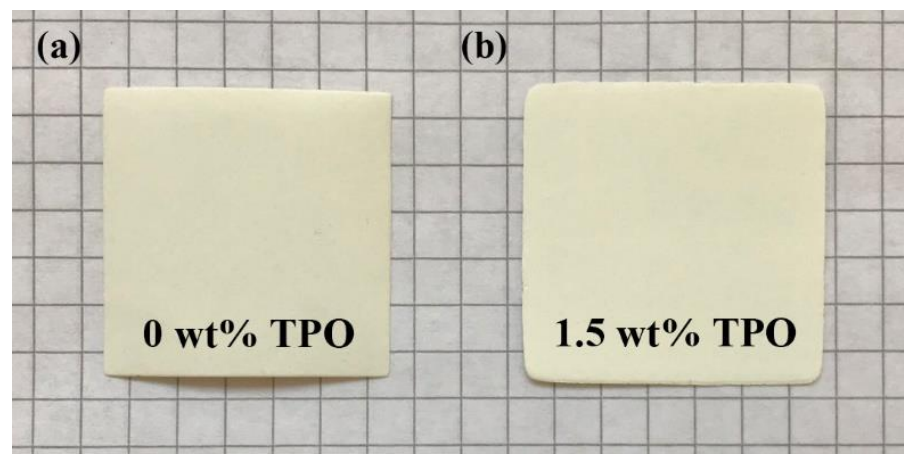

Figure 9: 3D printed piezoelectric composites. Fillers content: 10 vol\% of PZT in High-Temperature photopolymer resin. (a) No TPO added. Dimensions 30x30x0,2 mm; (b) With 1,5 wt\% of TPO added. Dimensions $32 \times 32 \times 0,2 \mathrm{~mm}$.

Temperature photopolymer but poor adhesion between ceramic inclusions and photopolymer was observed.

Suspension made of High-Temperature photopolymer and 10 vol\% of PZT was successfully printed with Prusa SL1 3D printer, while Formlabs Form 2 printer failed, mainly because it was not possible to adjust exposure time. The first layer adhesion problem on Prusa SL1 was solved by decreasing the first layer height to $20 \mu \mathrm{m}$, adjusting the first layer offset directly on the printer to $-0,150 \mu \mathrm{m}$ and increasing layer exposure time to 12 minutes. However, the first layer with a thickness of $120 \mu \mathrm{m}$ was achieved (set value was $20 \mu \mathrm{m}$ ), while consecutive layers were $50 \mu \mathrm{m}$ thick as was set in the slicing software.

An attempt to reduce exposure time required to cure a single layer was done by adding up to $4 \mathrm{wt} \%$ of photoinitiator TPO. At higher loadings, TPO was problematic to dissolve. The results showed a saturation of TPO around $0,5-1,5 \mathrm{wt} \%$. 3D printing of suspension with 1,5 wt $\%$ TPO revealed high over-exposure because of UV-light scattering by PZT particles and the need for further research.

\section{ACKNOWLEDGEMENTS}

The research project "Piezoelectric 0-0-3 Composites" is funded by DFG under the project number 389409970.

\section{REFERENCES}

[1] D. Yao, H. Cui, R. Hensleigh et al., "Achieving the Upper Bound of Piezoelectric Response in Tunable, Wearable 3D Printed Nanocomposites," Advanced Functional Materials, vol. 29, no. 42, p. 1903866, 2019.

[2] H. Cui, R. Hensleigh, D. Yao et al., "Threedimensional printing of piezoelectric materials with designed anisotropy and directional response," Nature materials, vol. 18, no. 3, pp. 234-241, 2019.

[3] C. Schmidleithner and D. M. Kalaskar, "Stereolithography," in 3D Printing, D. Cvetković, Ed., InTech, 2018.
[4] J. W. Halloran, "Ceramic Stereolithography: Additive Manufacturing for Ceramics by Photopolymerization," Annual Review of Materials Research, vol. 46, no. 1, pp. 19-40, 2016.

[5] J. H. Jang, S. Wang, S. M. Pilgrim et al., "Preparation and Characterization of Barium Titanate Suspensions for Stereolithography," Journal of the American Ceramic Society, vol. 83, no. 7, pp. 1804-1806, 2000.

[6] O. Dufaud and S. Corbel, "Stereolithography of PZT ceramic suspensions," Rapid Prototyping Journal, vol. 8, no. 2 , pp. 83-90, 2002.

[7] K. Kim, W. Zhu, X. Qu et al., "3D optical printing of piezoelectric nanoparticle-polymer composite materials," ACS nano, vol. 8, no. 10, pp. 9799-9806, 2014.

[8] Y. Yang, Z. Chen, X. Song et al., "Three dimensional printing of high dielectric capacitor using projection based stereolithography method," Nano Energy, vol. 22, pp. 414-421, 2016.

[9] K. Kim, J. L. Middlebrook, J. E. Chen et al., "Tunable Surface and Matrix Chemistries in Optically Printed (0-3) Piezoelectric Nanocomposites," ACS Applied Materials \& Interfaces, vol. 8, no. 49, pp. 33394-33398, 2016.

[10] P. Singh, L. S. Smith, M. Bezdecny et al., "Additive manufacturing of PZT-5H piezoceramic for ultrasound transducers," in IEEE International Ultrasonics Symposium (IUS), 2011: 18-21 Oct. 2011, Orlando, FL, USA, pp. 11111114, IEEE, Piscataway, NJ, 2011.

[11] H. Chabok, C. Zhou, Y. Chen et al., "Ultrasound Transducer Array Fabrication Based on Additive Manufacturing of Piezocomposites," in ASME/ISCIE 2012 International Symposium on Flexible Automation, pp. 433-444, [publisher not identified], [Place of publication not identified], 2012.

[12] X. Song, Z. Chen, L. Lei et al., "Piezoelectric component fabrication using projection-based stereolithography of barium titanate ceramic suspensions," Rapid Prototyping Journal, vol. 23, no. 1, pp. 44-53, 2017.

[13] O. Santawitee, S. Grall, B. Chayasombat et al., "Processing of printed piezoelectric microdisks: effect of PZT particle sizes and electrodes on electromechanical properties," Journal of Electroceramics, vol. 188, no. 1, p. 383, 2019.

[14] J. Cheng, Y. Chen, J.-W. Wu et al., "3D Printing of BaTiO3 Piezoelectric Ceramics for a Focused Ultrasonic Array," Sensors (Basel, Switzerland), vol. 19, no. 19, 2019.

[15] W. Chen, F. Wang, K. Yan et al., "Microstereolithography of KNN-based lead-free piezoceramics," Ceramics International, vol. 45, no. 4, pp. 4880-4885, 2019.

[16] J. Cha, J. W. Lee, B. Bae et al., "Fabrication and Characterization of PZT Suspensions for Stereolithography based on 3D Printing," Journal of the Korean Ceramic Society, vol. 56, no. 4, pp. 360-364, 2019.

[17] Z. Chen, Z. Li, J. Li et al., "3D printing of ceramics: A review," Journal of the European Ceramic Society, vol. 39, no. 4, pp. 661-687, 2019.

[18] S. Bodkhe and P. Ermanni, "Challenges in 3D printing of piezoelectric materials," Multifunctional Materials, vol. 2, no. 2, p. 22001, 2019. 
[19] C. Sun and X. Zhang, "Experimental and numerical investigations on microstereolithography of ceramics," Journal of Applied Physics, vol. 92, no. 8, pp. 4796-4802, 2002.

[20] M. L. Griffith and J. W. Halloran, "Freeform Fabrication of Ceramics via Stereolithography," Journal of the American Ceramic Society, vol. 79, no. 10, pp. 2601-2608, 1996.

[21] A. Badev, Y. Abouliatim, T. Chartier et al., "Photopolymerization kinetics of a polyether acrylate in the presence of ceramic fillers used in stereolithography," Journal of Photochemistry and Photobiology A: Chemistry, vol. 222, no. 1, pp. 117-122, 2011.

[22] Zachary Zguris, "How Mechanical Properties of Stereolithography 3D Prints are Affected by UV Curing," Formlabs, https://archive-media.formlabs.com/upload/HowMechanical-Properties-of-SLA-3D-Prints-Are-Affected-byUV-Curing.pdf.

[23] B. Steyrer, P. Neubauer, R. Liska et al., "Visible Light Photoinitiator for 3D-Printing of Tough Methacrylate Resins," Materials (Basel, Switzerland), vol. 10, no. 12, 2017.

[24] C. S. Sodré, P. P. A.C. Albuquerque, C. P. Isolan et al., "Relative photon absorption determination and the influence of photoinitiator system and water content on $\mathrm{C}=\mathrm{C}$ conversion, water sorption/solubility of experimental self-etch adhesives," International Journal of Adhesion and Adhesives, vol. 63, pp. 152-157, 2015.

[25] KMK Regulatory Services Inc, "Safety Data Sheet High Temp - Photoreactive Resin for Form 1+, Form 2," https://formlabsmedia.formlabs.com/datasheets/Safety_Data_Sheet_EN-EU__High_Temp.pdf.

[26] J. Bennett, "Measuring UV Curing Parameters of Commercial Photopolymers used in Additive Manufacturing," Additive manufacturing, vol. 18, pp. 203-212, 2017.

[27] M. Kneissl and J. Rass, eds., III-nitride ultraviolet emitters: Technology and applications, Springer, Cham, 2016.

[28] P. Elsner, "3D-Drucktechnologie - Grundlagen zur Herstellung polymerer Bauteile mit gradierten Werkstoffeigenschaften,” 2009. 